\title{
Les seuils du paradis
}

À l'instar de l'archipel caribéen, décrit dans l'avis au lecteur comme la «préface des Amériques » ou comme les Ant-isles, c'est-à-dire à la façon d'un espace qu'il faudrait nécessairement traverser afin d'atteindre la terre ferme, l'imaginaire du paradis se présente comme une sorte de seuil. Comment ce discours frontalier affecte-t-il l'organisation de la présentation du monde étranger? Dans un sens, sans s'inscrire directement dans la tradition de l'isolario, le livre lui-même ressemble à la géographie qu'il décrit, à un archipel, constitué d'îlots discursifs qui s'étendent à l'infini'. La comparaison n'est pas sans rappeler ce que Gérard Genette caractérise comme les «seuils» ou les «franges » d'un texte, à savoir le paratexte qui enveloppe et prolonge le texte, le transformant ainsi en livre ${ }^{2}$.

La relation de voyage du XVII ${ }^{\mathrm{e}}$ siècle est littéralement enveloppée de paratextes, dont la diversité excède largement celle de la liste proposée par Genette $^{3}$. Celles qui sont publiées en grand format, notamment, comme l'histoire de Du Tertre, en sont remplies à tel point qu'il semble que la paratextualité doive entrer dans la définition générique de la relation de voyage. La paratextualité effectue une sorte de compartimentation matérielle du discours et donne la priorité à une démonstration qui ne passe pas par l'échange oral, mais par la lecture. «Le lecteur », écrit Henri-Jean Martin, « cesse ainsi d'écouter en lui-même le discours reproduit sur la page : il regarde celle-ci et parfois la parcourt un peu à la manière d'une carte ${ }^{4}$ ». Le lecteur s'oriente lui-même dans les îlots textuels que le livre donne à voir.

1 Dans la mesure où la matière de l'Histoire générale des Antilles est l'archipel caribéen, elle prend forcément les traits d'un « recueil d'îles », racontant les îles les unes après les autres en faisant voir la singularité de chacune. Voir Frank Lestringant, Le Livre des îles : Atlas et récits insulaires de la Genèse à Jules Verne, Genève, Droz, 2002, ainsi que l'ouvrage qu'il a édité, Îles et insulaires $\left(X V I^{e}-X V I I^{e}\right)$ siècles, Paris, Presses de l'université Paris-Sorbonne, 2017. Éric Fougère, Les Voyages et l'ancrage : Représentation de l'espace insulaire à l'Âge classique et aux Lumières (1615-1797), Paris, L'Harmattan, 1995.

2 Gérard Genette, Palimpsestes : La littérature au second degré, Paris, Seuil, 1982, p. 7.

3 Sur l'importance du paratexte dans le genre viatique, voir Réal Ouellet, «Le paratexte liminaire de la relation : le voyage en Amérique», Cahiers de l'association internationale des études françaises, vol. $42 \mathrm{n}^{\circ}$ 1, 1990, p. 177-192.

4 Henri-Jean Martin, La Naissance du livre moderne : Les métamorphoses du livre français, Paris, Cercle de la Librairie, 200o, p. 328. 
Il ne s'agit pourtant pas uniquement de la disposition du savoir. Selon Genette, le paratexte indique la zone qui lie le texte au contexte et à une certaine politique. Cette dernière, qu'elle soit articulée par l'auteur ou par l'éditeur, Genette la renvoie à un sujet de discours. Cela dit, il est difficile, voire impossible, de démêler le paratexte auctorial du paratexte éditorial d'un ouvrage écrit sous mandat. Comme le soulignent Helen Smith et Louise Wilson dans Renaissance Paratexts, en s'appuyant sur les travaux d'Arthur Marotti, le paratexte à la Renaissance correspond à des « zones où des sources et des autorités multiples, et quelquefois concurrentes, sont la norme ${ }^{5}{ }$. Cette pratique paratextuelle se poursuit au XVII ${ }^{\mathrm{e}}$ siècle. La multiplication de ces seuils nous rappelle que la voix auctoriale du voyageur n'est pas seulement présente pour assurer la véracité du témoignage. Le paratexte garantit l'échange entre le texte et son lecteur, affichant une stratégie destinée à séduire le lecteur mondain, à satisfaire le savant avide d'information comme le simple amateur de curiosités, et enfin à convaincre le lecteur aventureux de se joindre à l'effort de colonisation des Îles. Moyennant quelques aménagements, la conception genétienne du paratexte en tant qu'il constitue entre « texte et hors-texte, une zone non seulement de transition, mais de transaction » s'avère donc éclairante pour la relation de voyage du XVII ${ }^{\mathrm{e}}$ siècle ${ }^{6}$. Zone transitoire, espace d'échange entre le narrateur et le narrataire, mais aussi le mandataire, le paratexte apparaît bien comme le «lieu privilégié d'une pragmatique et d'une stratégie, d'une action sur le public au service, bien ou mal compris et accompli, d'un meilleur accueil du texte et d'une lecture pertinente ${ }^{7}$ ».

Dans l'Histoire générale des Antilles, le paratexte ne se borne pas à l'espace préfaciel; il imprègne le texte. La structure thématique et architecturale de l'ouvrage fait en sorte que les seuils réapparaissent chaque fois qu'une nouvelle partie est introduite, et ils peuvent se glisser à l'intérieur même d'une partie pour préparer le lecteur à une séquence narrative particulière. Il y a donc des paratextes internes qui se multiplient. Non seulement chaque chapitre porte-t-il un titre indiquant le sujet traité, mais le sujet est lui-même introduit par des passages qui se distinguent de l'histoire racontée, fonctionnant comme autant de captationes benevolentiae. C'est surtout le paratexte interne qui est opératoire ici, dans la mesure où il met en valeur la façon dont l'exotisme se déploie dans une stratification de la relation de voyage, dont l'organisation

5 Helen Smith et Louise Wilson, Renaissance Paratexts, Cambridge, Cambridge University Press, 2011: « zones where multiple, and sometimes competing, authorities and sources are the norm », p. 8. Notre traduction.

6 Gérard Genette, Palimpsestes, p. 8.

7 Ibid. 
thématique permet au lecteur de commencer sa lecture n'importe où dans le livre et d'en reprendre ailleurs le fil, sur le mode de la consultation. Les cadres du récit invitent à cette sorte de lecture aléatoire; ils équilibrent l'ensemble, facilitent l'orientation du lecteur et se présentent comme des frontières textuelles que ce dernier pourra franchir comme il le veut.

\section{$\mathbf{1}$ \\ Encadrer les descriptions}

L'imaginaire paradisiaque se déploie précisément dans les paratextes internes ; c'est particulièrement le cas de celui que propose Du Tertre des Autochtones. Dans l'édition Langlois de 1654, il commence par prendre position contre les fausses idées répandues en Europe, selon lesquelles la zone torride serait un «séjour d'horreur et de supplices» et «le sauvage», un monstre. Il suffit de mentionner les Amérindiens pour qu'aussitôt « la plupart du monde se figure dans leurs esprits une sorte d'hommes Barbares, cruels, inhumains, sans raison, contrefaits, grands comme des geants, velus comme des ours: En fin, plustost des monstres que des hommes raisonnables [...] », (1654, tome II : 396) nous rappelle Du Tertre. Ce qui se présente ici comme une défense de l'Amérindien est en réalité un discours bien inséré dans le colonialisme français de l'époque, et dont le propos sert une critique de la libido dominandi des Espagnols, mais également de l'ambition impériale anglaise qui entre en concurrence avec la France pour posséder les Îles. Le propos du missionnaire changera d'ailleurs légèrement dans la deuxième édition, celle de 1667. L'exposition des préjugés contre les «sauvages » et leur monde est maintenant remplacée par une critique de la description des Amérindiens par de Rochefort qui, d'après Du Tertre, en présente une image largement embellie :

Ie prie pourtant le Lecteur de m'excuser, si ie ne les fais pas si polis que le sieur de Rochefort les a faits, en quelques endroits de son livre, puisque ie suivray en cela le sentiment de la pluspart de ceux qui les ont frequentez, qui m’ont protesté plusieurs fois, qu'ils ne les reconnoissoient plus dans la peinture qu'il en a faite. (1667, tome II : 355$)$

Le dominicain se conforme sur ce point à une pratique répandue dans les relations de voyage : il construit sa propre description en opposition à d'autres textes, afin de renforcer la crédibilité et l'originalité de sa relation ${ }^{8}$. Comme

8 Voir à ce sujet Réal Ouellet, «Le paratexte liminaire de la relation »: «Plutôt que concurrence mesquine, il faut voir là un trait spécifique de la relation de voyage : non seulement le 
il ne peut pas accuser de Rochefort d'être un voyageur de cabinet, il met en valeur la durée de son propre séjour aux Antilles pour mieux se distinguer de son rival et faire valoir ses observations longuement mûries. Le savoir que Du Tertre veut transmettre à son lecteur est présenté comme le fruit d'un processus d'observations, de réflexions, mais aussi de discussions avec d'autres savants. C'est cette expérience patiemment acquise qui constitue la base d'un savoir à la fois solide et modéré. Contre l'idéalisation aussi bien que contre la démonisation de l'Autochtone, Du Tertre offrirait donc une description plus fidèle de la réalité.

Le discours qui suit a cependant de quoi surprendre; dans un décor cette fois marqué par les stéréotypes, l'imaginaire du « sauvage » fait en effet irruption :

Or comme j'ay fait voir que l'air de la Zone torride est le plus pur, le plus sain \& le plus temperé de tous les airs, \& que la terre y est un petit Paradis tousiours verdoyant, \& arrousé des plus belles eaux du monde : il est à propos de faire voir dans ce traité, que les Sauvages de ces Isles sont les plus contens, les plus heureux, les moins vicieux, les plus sociables, les moins contrafaits, \& les moins tourmentez de maladies, de toutes les nations du monde. Car ils sont tels que la nature les a produits, c'est à dire, dans une grande simplicité \& naïfveté naturelle : ils sont tous égaux, sans que l'on connoisse presque aucune sorte de superiorité ny de servitude ; \& à peine peut-on reconnoistre aucune sorte de respect, mesme entre les parens, comme du fils au pere. Nul n'est plus riche, ny plus pauvre que son compagnon, \& tous unanimement bornent leurs desirs à ce qui leur est utile, \& precisément necessaire, \& méprisent tout ce qu'ils ont de superflu, comme chose indigne d'estre possedée. (1667, tome II : 356-57)

Cette partie, contenant l'histoire morale des Amérindiens, est sans doute le passage du livre qui a reçu le plus d'attention. Destiné à guider la lecture, ce discours introductif peint une toile de fond où se développera le portrait de l'Amérindien. On trouve ici une des rares occurrences du mot « paradis» dans l'Histoire générale des Antilles, et Du Tertre y déploie une série de topos paradisiaques. Au lieu de la description d'un site réel où habitent les Autochtones, on retrouve des références indirectes aux descriptions antérieures - on y reconnaît notamment l'essai Des cannibales, même si le texte de Montaigne n'est pas cité directement. Du Tertre ne se constitue donc pas dans cette introduction à la description morale comme un témoin oculaire, mais comme un lecteur; ce

suspense narratif doit tenir le lecteur en haleine, mais encore l'exotique qu'elle déroule sous les yeux du lecteur doit apparaître comme nouveau [...] », p. 19 . 
qu'il traite est l'image de l'Amérindien telle qu'elle est répandue en Europe. L'Autochtone est présenté dans un décor stéréotypé, calqué sur le modèle édénique, avec des références aux théories sur le climat et à la géographie des zones torrides.

Du Tertre opère ici entièrement à l'intérieur de la répétition discursive, et non dans le domaine référentiel normalement associé à la description dans une relation de voyage. Laccumulation des superlatifs, caractéristiques du discours paradisiaque et présentés sur un axe paradigmatique, nous éloigne de la rencontre réelle avec les Amérindiens. En renvoyant à un imaginaire détaché de la réalité décrite, les références textuelles censées renforcer la véracité du propos donnent au contraire au passage un aspect fragmentaire. Philippe Hamon l'a bien démontré : au lieu de représenter le monde comme un tout cohérent, ce type de liste d'attributs et de généralités contribue à faire éclater la réalité qu'elle est pourtant censée capturer9.

La description suscite ainsi un monde profondément paradoxal. Le pêle-mêle des attributs, des traits et des caractères des Autochtones ne peut pas s'harmoniser au sein de ce «petit Paradis tousiours verdoyant ». De la description stéréotypée et idéalisée de l'espace naturel s'ensuit donc un portrait tout aussi idéalisé que fragmentaire de la figure de l'Amérindien. En fait, le passage finit par inscrire celui-ci dans le décor d'une nature elle-même fantasmée. L'Autochtone mérite d'être décrit, car il est tout aussi particulier que la nature antillaise :

[...] en verité nos Sauvages ne soient Sauvages que de nom, ainsi que les plantes \& les fruits que la nature produit sans aucune culture dans les forests \& dans les deserts, lesquelles quoy que nous les appelleions Sauvages, possedent pourtant les vrayes vertus \& les proprietez dans leur force \& dans leur entiere vigueur, que bien souvent nous corrompons par nos artifices, \& alterons beaucoup, lors que nous les plantons dans nos jardins. (1667, tome II : 356 ; nous soulignons.)

Tandis que la différence entre les Antilles et l'Europe est bien mise en valeur, l'analogie entre les plantes sauvages et les Autochtones fait de l'espace naturel antillais un ensemble homogène sans rupture de continuité entre les hommes

9 Philippe Hamon rappelle que le descriptif ne renvoie pas à un référent, mais à une mise en ordre, à une mise en classement sémiologique. Du descriptif, Paris, Hachette, 1993, p. 6o. Il est difficile de ne pas penser à Bakhtine, qui analyse un style insulaire marqué par la syncope, la parataxe ou la phrase en éventail, typique du paysage idyllique. Voir Mikhaïl Bakhtine, Esthétique et théorie du roman, Paris, Seuil, 1978, p. 254. 
et la nature. On se croirait dans une description préromantique de ce rapport, qui serait impensable s'il était question ici de l'homme européen et de son inscription dans la nature. La mise en ordre humaine ne reflète plus l'harmonie divine - c'est la nature sauvage qui en devient le miroir, et cette conception de la nature est intéressante pour un texte écrit au milieu du XVII ${ }^{\mathrm{e}}$ siècle, d'autant plus qu'elle n'est pas très illustrative pour comprendre l'idée générale de la nature dans l'Histoire générale des Antilles, comme nous le verrons. Sauvage, le paysage ne ressemble ni à un décor façonné par l'homme ni à l'idée d'une nature parfaite, vierge, reflet idéal de la création divine. Ici, c'est l'Amérindien, le «Sauvage» dans son habitat naturel, qui vit comme les plantes produites «sans aucune culture», et qui seul peut rappeler la création de Dieu. Si sa nature semble ainsi parfaite, sa culture apparaît bien sûr perfectible aux yeux du missionnaire. Par exemple, «le front plat \& le nez camus» des Amérindiens «ne [proviennent] pas d'un défaut de nature, mais de l'artifice de leurs meres, qui mettent leurs mains sur le front de leurs enfans pour l'aplatir \& l'élargir tout ensemble $[\ldots]^{10}$ ». $(1667$, tome II : $357-58)$ Mais à la différence du propos de Montaigne, la critique de la soumission des Caraïbes à un ordre « civilisé » s'articule ici à un projet politique colonial spécifique. La culture caribéenne est mauvaise dans la mesure où il lui manque la religion chrétienne, mais aussi en raison de l'influence pervertissante d'une autre culture dominante, celle apportée par les colons français: « ie puis dire avec verité, que si nos Sauvages sont plus ignorans que nous, qu'ils sont beaucoup moins vicieux, voire mesme qu'ils ne scavent presque de malice que ce que nos François leur en apprennent ${ }^{11}$ ». $(1667$, tome II : 358$)$

La partie historique qui précède la description a déjà exposé les effets de cette influence culturelle qui dénature l'Amérindien. Se construit ainsi un discours de domination par bienveillance qui se donne pour but de sauvegarder

10 Frank Lestringant fait remarquer que le nez écrasé est dans la littérature de voyage du $\mathrm{XVI}^{\mathrm{e}}$ siècle la «caractéristique des peuples inférieurs appelés à être dominés », mais que cette malédiction ne concerne pas les Amérindiens du Brésil : « Lécrasement du nez n'est pas chez eux congénital, mais le fruit d'un usage aberrant. Les Cynocéphales ne sont pas tels "naturellement, mais artificiellement". » Le Cannibale, p. 53. Du Tertre semble partager ce point de vue.

11 On pourrait à ce propos s'étendre sur une thématique répandue des récits de voyage qui est celle de la religion primitive des « sauvages ». Bernard Tocanne identifie un schéma typique aux récits de missionnaires, qui esquisse une religion primitive corrompue par des «prêtres imposteurs annonçant une religion prétendue révélée ». Voir L'Idée de nature en France dans la seconde moitié du XVII e siècle, p. 273. Comme le thème de la mission même est relativement absent de l'ouvrage de Du Tertre, ce schéma ne s'impose pas. Par contre, notre missionnaire évoque souvent l'influence négative des «imposteurs» européens selon un schéma semblable à celui qu'identifie Tocanne. 
la nature de l'Autochtone tout en lui refusant toute transformation et en niant son inscription dans une temporalité historique. Ce qui est déploré ici, c'est le monde vierge d'avant l'exploitation coloniale, perdu à jamais.

La position du discours introductif qui entoure la description laisse comprendre que ce tableau s'élabore à partir d'autres textes et n'émane pas de l'observation directe. Il est teinté d'une nostalgie qui, selon Jean Delumeau, prend son sens dans le cadre d'une époque classique où l'on rêve de retrouver l'âge d'or parce que l'on a abandonné l'idée que l'Éden terrestre existe vraiment ${ }^{12}$. Ces renvois implicites à un topos contemporain montrent bien que le passage crée un espace intertextuel qui introduit une distance par rapport au monde raconté. La description anthropologique se détache ainsi étrangement du monde qu'elle se propose de décrire. C'est un éloignement conceptuel qui s'impose pour mieux rapprocher le lecteur de l'étranger. À travers les références au paradis que tout lecteur peut reconnaître se construit un espace homogène sur lequel Du Tertre pourra ensuite déployer ses tableaux des mœurs antillaises.

Cette fonction paradoxale du paratexte interne qui crée une distance par rapport à la réalité pour mieux nous en rapprocher apparaît avec plus de clarté encore dans les estampes incluses dans l'édition de 1667, où l'idéalisation d'une nature tropicale paradisiaque prend un autre tour. Dans la figuration des Amérindiens que proposent les illustrations de Leclerc, ce n'est pas l'exubérance tropicale qui règne, mais l'harmonie, ce qui nous resitue dans le cadre du jardin que Du Tertre vient pourtant de réfuter comme étant étranger à l'Amérindien. C'est, pour ainsi dire, la version visuelle classique du «bon Sauvage », dont la représentation rompt radicalement avec les illustrations de l'Amérique du siècle précédent, largement dominées par l'imaginaire de la férocité cannibale ${ }^{13}$.

La gravure de Leclerc est fortement architecturée et centralisée ; elle impose un filtre culturel français à la présentation de la nature antillaise : un homme et une femme posent à côté d'un papayer franc. L'arbre a une double fonction ; il sert de support à la description moraliste que présente le texte - l'espèce de l'arbre est précisée avec un renvoi à la page où se trouve la description - et il

12 Jean Delumeau, Une histoire du paradis: Le jardin des délices, Paris, Fayard, 1992, p. 152-53. Cette nostalgie coïncide avec la mise en valeur du jardin. Voir aussi Jean Céard, «Avant-propos », Voyager à la Renaissance : «À la différence du Barbare, que la tradition humaniste et l'antiquité avaient en quelque sorte assimilé en lui conférant un statut au sein d'une dialectique linguistique et socio-culturelle de la barbarie et de la civilisation, le Sauvage est le primitif qui vit au sein d'une nature exubérante et vierge, ignorant les lois les plus élémentaires de la société - et surtout de la société urbaine, modèle européen de la société et de la sociabilité [...] », p. 14-15.

13 Voir à ce sujet Frank Lestringant, Le Théâtre de la Floride. 


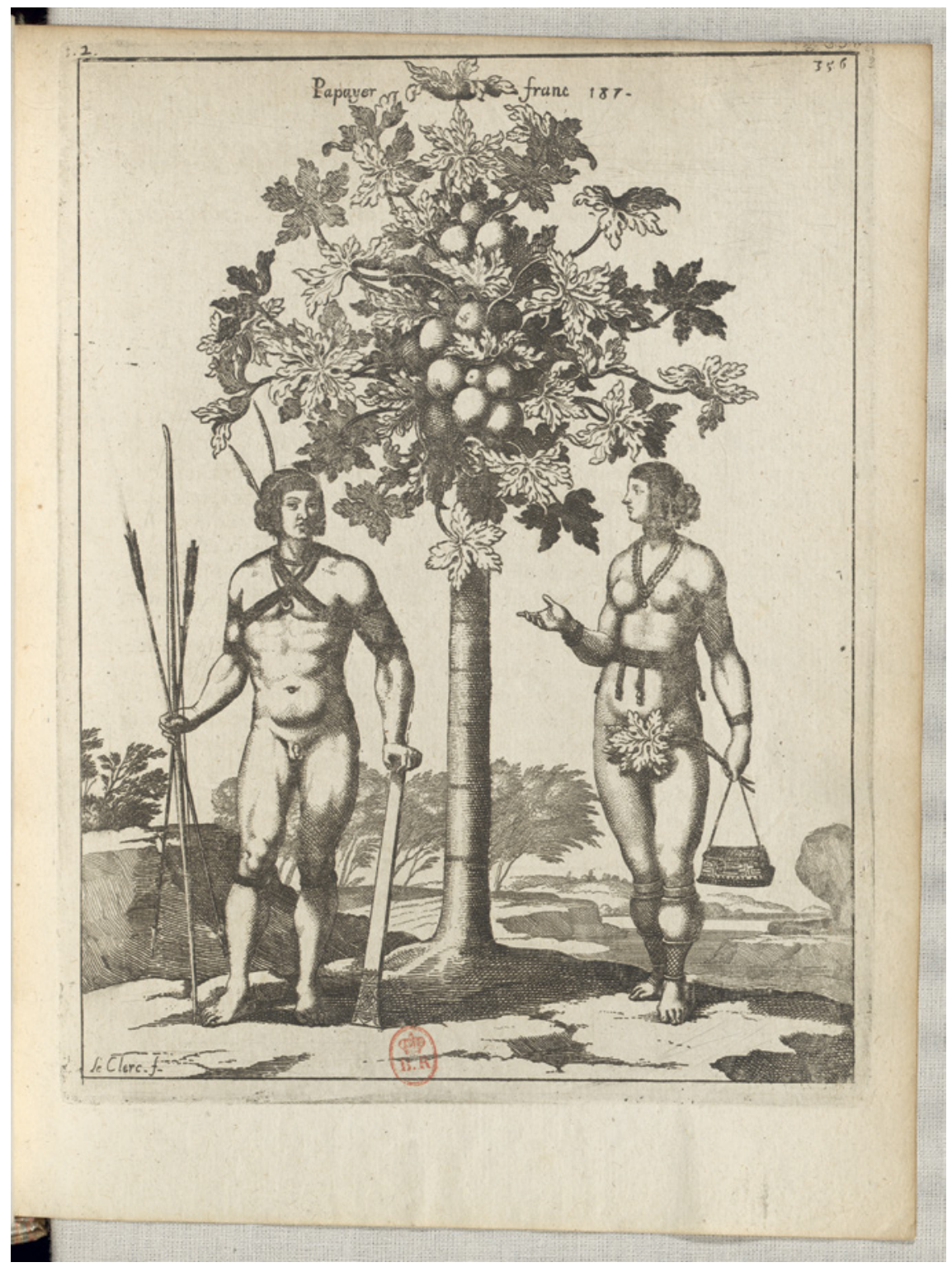

ILLUSTRATION 3 Papayer franc, homme et femme sauvages, Sébastien Leclerc

SOURCE GALLICA.BNRF.FR / BIBLIOTHÈQUE NATIONALE DE FRANCE, DOMAINE PUBLIC 
fonctionne en même temps comme support visuel à l'illustration des hommes. La femme couvre son sexe d'une feuille de papayer alors qu'elle se tourne vers l'homme de l'autre côté de l'arbre; l'allusion à Ève évoque ainsi le débat sur le statut de ces infidèles, dans la théologie de la création et de la chute. L'homme en revanche est nu, mais sa pose en contrapposto relie cette nudité à celle de la statuaire antique autant qu'à l'imaginaire de la sauvagerie. Seuls les objets une sorte de hache, des flèches, des ornements (colliers, ceintures, bracelets autour des bras et des genoux, plumes) - et une corbeille portée par la femme permettent d'identifier l'origine ethnique des modèles. Les traits sont plutôt européens, la femme a le profil d'un Botticelli, et leurs corps musclés, grands et équilibrés correspondent à l'idéal classique; ils ne portent pas les cheveux longs, et seule une petite plume évoque la couleur locale ${ }^{14}$. L'ensemble l'arbre, l'homme et la femme - forme presque une voûte, rappelant ainsi l'idée d'un seuil vers ce monde des Caraïbes que l'arrière-plan de l'illustration nous laisse seulement deviner. Le papayer tient lieu d'élément architectural de l'illustration et joue par là le rôle de charnière entre la nature édénique sauvage et le jardin où les Français auraient «cultivé » ces «plantes sauvages», pour reprendre la formulation de Du Tertre; s'il est plus cultivé que sauvage, l'arbre porte toutefois des fruits mûrs et succulents en abondance, rappelant ainsi le printemps éternel qui règne dans ce paradis «tousiours verdoyant» esquissé plus tôt dans l'Histoire générale des Antilles.

\section{Retour à la fiction}

Entre le passage introductif idéalisé et le contenu même de la section sur les Amérindiens, on observe un décalage au niveau de l'organisation du texte et de l'écriture elle-même, dans la mesure où le style de l'introduction est répétitif, tendant vers la généralisation, alors que la description qui suit est dominée par l'observation, souvent personnelle. Quant à l'illustration, elle obéit évidemment à d'autres règles : en tant que représentation de l'autre, elle joue à la fois sur l'étrangeté, par l'introduction d'objets exotiques américains, et sur les exigences de la représentation de la belle nature ${ }^{15}$. Le travail du graveur

14 Comme François de Dainville le rappelle à propos des gravures dans les œuvres de Thevet: «La beauté de ses sauvages, dont les anatomies et les physionomies dénotent l'influence de l'antiquité et évoquent les figures de l'école de Carrache, ont plus fait pour établir le prestige de "l'homme de la nature" que les éloges de vingt relations », La Géographie des humanistes, p. 16o.

15 François Moureau, Le Théâtre des voyages: Une scénographie de l'âge classique, Paris, Presses de l'université Paris-Sorbonne, 2005, p. 38. 
témoigne ainsi de ce que l'illustration du voyage commence alors à se formaliser et, comme l'a démontré François Moureau, «fait l'économie d'une "poétique de l'espace" que condamne le principe de transposition objective ${ }^{16}$ ». Les gravures de Leclerc ne tentent pas de reproduire mimétiquement le réel; elles sont indicatives d'une certaine conception du monde étranger.

Bien que la représentation visuelle soit soumise à d'autres codes que le passage introductif idéalisé, ces deux expressions partagent un même rapport marginal à l'histoire racontée par la relation. On pourrait dire avec Roland Le Huenen qu'elles ont une autre « relation formelle à l'ordre du temps ${ }^{17}{ }^{17}$. Mais si Le Huenen repère une distinction entre l'ordre temporel de la narration et celui de la description, la manière dont le relateur encadre les différentes parties de son ouvrage ajoute une complication temporelle au sein même du régime descriptif chez Du Tertre. Car les discours introductifs opèrent un retour à la fiction, donc à une autre temporalité que celle que l'on retrouve dans le corps du texte. L'imaginaire paradisiaque, façonné à l'image du sauvage, introduit une pause dans la narration et refuse à l'Amérindien la mobilité aussi bien que la contemporanité, comme si la société autochtone existait dans une temporalité différente à celle de la société des habitants français. Il y aurait déjà chez Du Tertre alors ce que Johannes Fabian identifie comme le schéma temporel qui régit le fondement de l'anthropologie, à savoir un denial of coeavalness ${ }^{18}$. L'espace prend la relève sur le temps et marque le retour à une idéalisation de la nature qui affecte aussi la conception de l'homme autochtone. Le rêve de l'âge d'or propre à la pensée de l'époque est ici empreint d'un certain primitivisme: Du Tertre esquisse les traits d'un paysage statique qui tend vers le pictural et dont l'organisation visuelle ressort avec force. L'introduction ne progresse ni selon l'ordre chronologique de l'histoire de l'établissement de la colonie ni au rythme des descriptions des Autochtones, qui inscrivent un savoir dans le récit. Elle se construit sur une temporalité stagnante, idyllique, entièrement intertextuelle. Dans cet espace, le portrait stéréotypé de l'Amérindien permet au relateur à la fois d'assouvir les attentes du public et de maintenir à distance l'idéalisation qu'il vient de créer.

L'histoire morale semble ainsi soumise à deux régimes d'écriture différents : un régime littéraire et un régime factuel. Le régime littéraire ou poétique domine le paratexte interne, tandis que le factuel régit le corps de la description et de la narration qui en découlent. Dès que l'on avance dans la description

\footnotetext{
$16 \quad$ Ibid., p. 41.

17 Roland Le Huenen, «Le récit de voyage : l'entrée en littérature », p. 48.

18 Johannes Fabian, Time and the Other: How Anthropology Makes its Object, New York, Columbia University Press, 2014 [1983], p. 17.
} 
ou que l'on se reporte à la partie historique de l'ouvrage, on remarque que les énumérations d'idées et d'images préconçues se font plus rares, même si certains stéréotypes émergent ici également. Les scènes d'échanges avec les Autochtones sont par exemple émaillées de mentions de vieillards qui seraient âgés de plus de 150 ans, exagération qui relève du registre paradisiaque. Mais en général, l'histoire racontée nuance les généralisations stéréotypées, autant négatives que positives, en prêtant attention aux détails et en incluant des anecdotes que Du Tertre tire de ses propres rencontres avec les Amérindiens. Il convient ici de suivre Marie Maclean lorsqu'elle distingue le texte du paratexte en s'appuyant sur la théorie des actes locutoires, afin de préciser la différence de niveau qui existe entre le corps textuel d'un livre et le paratexte:

Les paratextes impliquent une série d'actes illocutoires de premier ordre dans lesquels l'auteur, l'éditeur ou le préfacier utilisent fréquemment des performatifs directs. Ils informent, persuadent, conseillent ou même exhortent et dirigent le lecteur ${ }^{19}$.

Même si, dans le cas de la relation de voyage, ni le texte ni le paratexte n'appartiennent pleinement à un régime fictionnel, le propos de Maclean reste utile ici. L'énonciation diffère dans les parties introductives et dans la narration. Les séquences textuelles qui ouvrent certains chapitres font clairement entendre une voix auctoriale qui cherche à instruire et à préparer son lecteur, tandis que la voix narrative rapporte les événements historiques ou les anecdotes du séjour. Autrement dit, le passage introductif ne contient pas une description de ce que le missionnaire aurait $v u$, mais offre un tableau répondant aux attentes du public.

Le passage qui introduit la description anthropologique, notamment, a une fonction qui évoque l'ut pictura poesis, au sens où sa référence n'est pas ce qui se présente aux yeux de l'observateur ${ }^{20}$. Puisque les descriptions visuelles et textuelles sont à ce point formalisées, la nature qu'elles donnent à voir se

19 Marie Maclean, «Pretexts and Paratexts: The Art of the Peripheral », New Literary History, vol. 22, 1991, p. 274. Aussi cité dans Smith et Wilson, Renaissance Paratexts, p. 10. «The paratexts involve a series of first order illocutionary acts in which the author, the editor, or the prefacer are frequently using direct performatives. They are informing, persuading, advising, or indeed exhorting and commanding the reader. » Notre traduction.

20 Reprenons à ce propos la définition de la description par Philippe Hamon : « un savoir (de mots, de choses) est non seulement un texte déjà appris, mais aussi un texte déjà écrit ailleurs, et la description peut donc être considérée toujours, peu ou prou, comme le lieu d'une réécriture, comme un opérateur d'intertextualité ; de-scribere, rappelons-le, étymologiquement, c'est écrire d'après un modèle ». Du descriptif, p. 48. 
détache de la réalité et s'approche d'une représentation, construite sur l'effacement de tout objet original, authentique. Contrairement à ce que l'on peut croire, une telle géographie imaginaire ne nuirait pas à la constitution d'un savoir. L'historien obéit aux règles, comme le fait l'artiste ou l'écrivain classique cherchant à donner à la nature une forme intelligible. Les stéréotypes tiennent lieu de fond commun, universel, sur lequel le relateur, comme le poète et le peintre, peut communier avec le Vrai ${ }^{21}$.

Les passages introductifs s'imposent donc, mais selon une autre discursivité que celle de l'histoire racontée. Cela explique pourquoi Du Tertre, comme tant d'autres, puise aussi bien dans les sources textuelles que dans les sources iconographiques disponibles et situe le lecteur au cœur du lieu commun d'une nature sauvage qui n'est pas sans rappeler les paysages bucoliques ${ }^{22}$. S'attendant à trouver ce type de paysage, le lecteur de l'époque identifiait son statut particulier dans le texte et pouvait lire cette introduction comme une captatio benevolentiae à cette sous-partie descriptive consacrée aux Amérindiens des Îles plutôt que comme un passage mimétique ou référentiel ${ }^{23}$. À cet égard, il faut également nuancer le propos de Bernard Tocanne qui, à la lecture précisément du passage introductif à la description des Amérindiens chez Du Tertre, suggère qu' « [i]nterprétant leur expérience à travers leurs souvenirs littéraires de lâge d'or ou la tradition théologique de l'Éden, les Européens ont cru retrouver la nature ${ }^{24} \gg$. En fait, cette redécouverte de la nature n'est que formelle; elle doit être interprétée à la lumière de la structure complexe du livre et de la variété des fonctions assumées par les différents discours qui coexistent dans la relation. Le passage introductif ne s'appuie pas sur la logique du discours savant ou de la philosophie; il procède à une idéalisation comparable à celle que l'on pourrait retrouver dans la représentation d'un paysage bucolique dans un roman.

La relation est donc construite sur des discours contradictoires qui se déploient sur des plans narratifs distincts. Ils peuvent coexister grâce aux frontières établies dans le récit même par le truchement des parties introductives, qui jouent à la fois le rôle d'intermédiaires et de démarcations. Ainsi, le discours paradisiaque tient-il ensemble la partie historique et la partie descriptive : d'un côté, il appartient à une réflexion philosophique et théologique; de l'autre, il sert de décor dans certains endroits précis de la relation. En gros, il

\footnotetext{
21 Bernard Tocanne, L'Idée de nature en France dans la seconde moitié du XVII e siècle, p. 324.

22 Peter Hulme, Colonial Encounters, p. 32.

23 Voir Helen Smith et Louise Wilson, Renaissance Paratexts, p. 4-5.

24 Bernard Tocanne, L'Idée de nature en France dans la seconde moitié du XVII e siècle, p. 199. Voir aussi l'analyse de Christian Marouby qui va dans le même sens, inscrivant les propos de Du Tertre dans une tradition primitiviste. Utopie et primitivisme, p. 109.
} 
remplit la fonction de médiateur, et ce grâce à son détachement par rapport à la diégèse viatique, c'est-à-dire par rapport à l'histoire de l'établissement et à ce que le missionnaire a vu et vécu aux Îles. Si l'on prend en considération la cohérence même de l'oscillation qui parcourt la relation, il devient difficile de conclure qu'il s'agit d'une simple contradiction ou de la faiblesse d'un relateur qui ne se déciderait pas à prendre parti. L'image idéalisée de l'Éden sauvage trouve sa justification logique dans l'organisation interne du texte. Plutôt que de voir dans les passages itératifs une reproduction mimétique de la nature et de l'Amérindien, il faut donc conclure que le relateur produit une géographie transitoire, comme si le texte cherchait partout à faciliter le passage au monde étranger. Une fois le seuil vers l'étranger franchi, l'auteur peut s'éloigner du tableau idéalisé et itératif afin de livrer au lecteur sa version de ce qu'il a d'abord lu, avant de l'observer aux Îles. C'est cette transition que semble mobiliser l'exotisme: son objectif principal n'est pas de doter un espace réel d'attributs exotiques, mais bien de créer un passage vers un monde lointain. 